\title{
SUPPRESSING SIDE-LOBES OF LINEAR PHASED ARRAY OF MICRO-STRIP ANTENNAS WITH SIMULATION-BASED OPTIMIZATION
}

\author{
Sławomir Koziel, Stanislav Ogurtsov, Adrian Bekasiewicz
}

Reykjavik University, Engineering Optimization \& Modeling Center, Menntavegur 1, 109 Reykjavik, Iceland ৯koziel@ru.is, +3545996376,stanislav@ru.is, bekasiewicz@ru.is)

\begin{abstract}
A simulation-based optimization approach to design of phase excitation tapers for linear phased antenna arrays is presented. The design optimization process is accelerated by means of Surrogate-Based Optimization (SBO); it uses a coarse-mesh surrogate of the array element for adjusting the array's active reflection coefficient responses and a fast surrogate of the antenna array radiation pattern. The primary optimization objective is to minimize side-lobes in the principal plane of the radiation pattern while scanning the main beam. The optimization outcome is a set of element phase excitation tapers versus the scan angle. The design objectives are evaluated at the high fidelity level of description using simulations of the discrete electromagnetic model of the entire array so that the effects of element coupling and other possible interaction within the array structure are accounted for. At the same time, the optimization process is fast due to SBO. Performance and numerical cost of the approach are demonstrated by optimizing a 16-element linear array of microstrip antennas. Experimental verification has been carried out for a manufactured prototype of the optimized array. It demonstrates good agreement between the radiation patterns obtained from simulations and from physical measurements (the latter constructed through superposition of the measured element patterns).
\end{abstract}

Keywords: linear antenna array, micro-strip antenna array, phased antenna array, antenna optimization, antenna array optimization, simulation-based optimization, surrogate model.

C) 2016 Polish Academy of Sciences. All rights reserved

\section{Introduction}

Design of phased antenna arrays is a challenging task due to a large number of designable parameters and, in particular, the necessity of accounting for element coupling, element environment, feeding, finite size of substrate, radome, etc. [1,2]. Excitation of a phased antenna array with phase-only tapers is attractive from the implementation standpoint [3-5]. Phase-only synthesis has been performed using numerical optimization [3-5] of suitably defined objective functions (e.g. the side-lobe level). Popular tools for phased array pattern synthesis include population-based metaheuristics [6-8]. Although these methods have proven to be useful for handling certain challenges (e.g. multiple local optima), they are characterized by a tremendous computational cost, which can be prohibitive in the context of simulation-driven design accounting for element coupling, emission of surface waves, and the array environment. Consequently, design of phased array antennas (especially with phase-only excitation tapers) is normally performed through optimization of the associated array factor [3-8] and analytical models accounting for coupling (see, e.g. [3]).

Scanning can lead to degradation of array performance, e.g. rising side-lobes, grating lobes, scan blindness, etc. [1]. This issue can be partially addressed by using rather simplified Electromagnetic (EM) [8] or circuit [9] models of the antenna array at hand. Systematic handling of this problem requires re-optimization of phase tapers for a range of scan angles 
of interest. Unfortunately, this is virtually prohibitive - due to an excessive computational cost when applied at the level of accurate EM discrete model of the entire array aperture and using conventional numerical optimization methods (e.g. gradient-based or metaheuristic algorithms). On the other hand, Surrogate-Based Optimization (SBO) methodologies [10] can be used for reliable antenna array designs accounting for different EM interactions and structural details, in particular with phase-only tapers with acceptable numerical costs [11, 12].

In this paper, we extend the approach of [11] so that the phase-only excitation of a linear phased antenna array of interest is optimized for a reduced Side-Lobe Level (SLL) for selected scan angles at the operational frequency. For each element, the excitation within the scan sector is obtained via interpolation of the optimal values. The design procedure is based on expedited optimization of the radiation pattern exploiting a suitably corrected array factor model. It is demonstrated through the design of a linear array comprising sixteen individually fed microstrip antennas. The element and feed geometry as well as the optimal excitations are obtained at the high-fidelity level of EM descriptions and with reasonable computational costs. The fully simulated radiation pattern of the optimal design is also compared with the radiation pattern evaluated using sixteen measured radiation patterns of the embedded array elements.

\section{Optimization methodology}

Optimization of the EM-simulated antenna array model $\boldsymbol{R}_{f}$ can be accelerated by exploiting the SBO paradigm $[10,11]$ and using the analytical array factor model $\boldsymbol{R}_{a}$ (with ideal isotropic radiators) as an underlying low-fidelity array model. We have two objectives: (i) simultaneous minimization of SLL and centering the reflection responses on the operating frequency $f_{0}$, and (ii) obtaining phase excitation tapers which ensure minimal SLL in the process of beam scanning.

\subsection{Element optimization}

Here, we optimize the EM model of the radiating element, $\boldsymbol{R}_{e f}\left(\boldsymbol{x}_{g}\right)$, where $\boldsymbol{x}_{g}$ represents geometry parameters. The optimization process uses a corrected coarse-discretization model of the element, $\boldsymbol{R}_{e c}$. Due to the narrow-band reflection response of the antenna array, the best way to align $\boldsymbol{R}_{e c}$ with $\boldsymbol{R}_{e f}$ is frequency scaling $F(\omega)=\alpha_{0}+\alpha_{1} \omega$ [13], where $\alpha_{0}$ and $\alpha_{1}$ are the parameters to be determined. The frequency scaled model is defined as:

$$
\boldsymbol{R}_{e c . F}(\boldsymbol{x})=\left[R_{e c}\left(\boldsymbol{x}_{g}, F\left(\omega_{1}\right)\right), \ldots, R_{e c}\left(\boldsymbol{x}_{g}, F\left(\omega_{m}\right)\right)\right]^{T},
$$

where: $\boldsymbol{R}_{e c}\left(\boldsymbol{x}_{g}\right)=\left[R_{e c}\left(\boldsymbol{x}_{g}, \omega_{1}\right), \ldots, R_{e c}\left(\boldsymbol{x}_{g}, \omega_{m}\right)\right]^{T}$ is the reflection coefficient at the frequencies $\omega_{j} ; j=$ $1, \ldots, m ; \alpha_{0}, \alpha_{1}$ are obtained as:

$$
\left[\alpha_{0}^{(i)}, \alpha_{1}^{(i)}\right]=\arg \min _{\left[\alpha_{0}, \alpha_{1}\right]} \sum_{k=1}^{m}\left[R_{e f}\left(\boldsymbol{x}_{g}, \omega_{k}\right)-R_{e c}\left(\boldsymbol{x}_{g}, \alpha_{0}+\alpha_{1} \omega_{k}\right)\right]^{2} .
$$

The optimized element design is found as:

$$
\boldsymbol{x}_{g}^{o p t}=\arg \min _{\boldsymbol{x}_{g}} U_{S 11}\left(\boldsymbol{R}_{e c . F}\left(\boldsymbol{x}_{g}\right)\right),
$$

where $U_{S 11}$ is the objective function defined as the reflection coefficient at the operating frequency $f_{0}$. In practice, up to three iterations (3) are sufficient to find the optimum of $\boldsymbol{R}_{\text {ef }}$ (with the scaling (1), (2) repeated at the beginning of each iteration).

\subsection{Correction of the Array Factor Model}

Discrepancies between the models, used to evaluate the array radiation pattern, can be reduced by applying a suitable response correction function $D(\theta)$. It is defined at the initial 
design (here, with all excitation phases $\boldsymbol{x}_{p}$ equal to zero) as follows. Let $p_{f}^{k}=\left[\theta_{f}^{k} r_{f}^{k}\right], k=1, \ldots$, $K_{f}$, be $K_{f}$ points extracted from the nominal $\boldsymbol{R}_{f}$ response (cf. Fig. 1) where $\theta_{f}{ }^{k}$ is the angle and $r_{f}{ }^{k}$ is the corresponding achieved gain value, for $\theta=0,+180$ degrees as well as all local realized gain maxima in the range $[0,+180]$ degrees; $p_{a}{ }^{k}=\left[\theta_{a}{ }^{k} r_{a}{ }^{k}\right], k=1, \ldots, K_{a}$, denote similar points extracted from the $\boldsymbol{R}_{a}$ response (here $\left.K_{a}=K_{f}\right)$. $D(\theta)$ is defined as:

$$
D(\theta)=E_{f}(\theta)-E_{a}(\theta)
$$

with $E_{f}(\theta)=F\left(\left[\theta_{f}^{1} \ldots \theta_{f}^{K_{f}}\right],\left[r_{f}^{1} \ldots r_{f}^{K_{f}}\right], \theta\right)$, where $F(X, Y, x)$ is the interpolation of the data value vector $Y$ (defined on a discrete argument set $X$ ) onto $x$. A similar definition holds for $E_{a}(\theta)$. Fig. 1 shows the plot of $D(\theta)$ for the linear array, extracted from the responses $\boldsymbol{R}_{f}$ and $\boldsymbol{R}_{a}$.

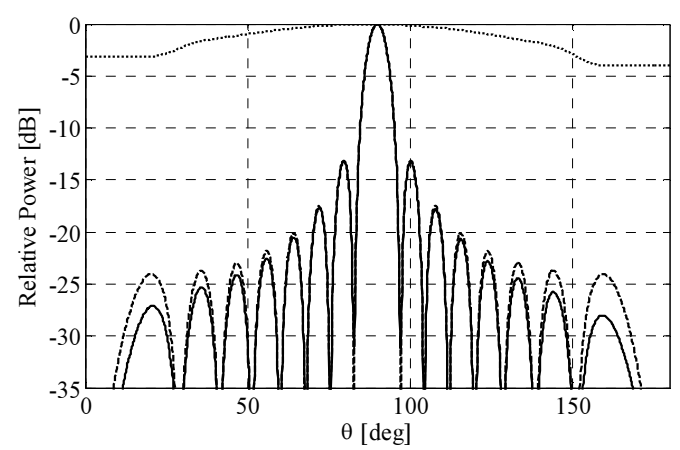

Fig. 1. The Power pattern of the 16-element array antenna in the E-plane at $10 \mathrm{GHz}$ at the initial design: (-) the EM model $\boldsymbol{R}_{f}$, and (- - ) the analytical array factor model $\boldsymbol{R}_{c} ;(\cdots \cdots)$ the correction function $D$ extracted from $\boldsymbol{R}_{f}$ and $\boldsymbol{R}_{a}(c f$. (4)).

The surrogate model $\boldsymbol{R}_{s}$ is defined using $D(\theta)$ as follows:

$$
\boldsymbol{R}_{s}\left(\boldsymbol{x}_{p}, \theta\right)=\boldsymbol{R}_{a}\left(\boldsymbol{x}_{p}, \theta\right)+D(\theta),
$$

where $\boldsymbol{x}_{p}=\left[\begin{array}{llll}p_{1} & p_{2} & \ldots & p_{N}\end{array}\right]$ is the phase excitation. Note, that $D$ generally depends on the array geometry (in particular, on $\boldsymbol{x}_{g}$ ) and the current phase taper $\boldsymbol{x}_{p}^{\text {curr }}$, so we have $D(\theta)=D\left(\boldsymbol{x}_{g}, \boldsymbol{x}_{p}^{\text {curr }}, \theta\right)$. Consequently, we write $\boldsymbol{R}_{s}\left(\boldsymbol{x}_{p} ; \boldsymbol{x}_{g}, \boldsymbol{x}_{p}^{\text {curr }}, \cdot\right)=\boldsymbol{R}_{a}\left(\boldsymbol{x}_{p}, \cdot\right)+D\left(\boldsymbol{x}_{g}, \boldsymbol{x}_{p}{ }^{\text {curr }}, \cdot\right)$ to indicate its dependence on both $\boldsymbol{x}_{g}$ and $\boldsymbol{x}_{p}^{\text {curr }}$.

\subsection{Array design for scanning}

At this stage, we search for a phase excitation taper ensuring minimum SLL for a given scan angle $\alpha$. We start from optimizing the array for $\alpha=0$, using the surrogate model (5) as follows:

$$
\boldsymbol{x}_{p}^{o p t}=\arg \min _{\boldsymbol{x}_{p}} U_{S L L}\left(\boldsymbol{R}_{s}\left(\boldsymbol{x}_{p}, \boldsymbol{x}_{g}\right)\right),
$$

where $U_{S L L}$ evaluates the SLL for any given $\boldsymbol{x}_{p}$. The random-search-initialized gradient-based algorithm [16] is used at this point. Because of mutual coupling within the array, the active reflection responses of the EM-simulated array at $\boldsymbol{x}_{p}{ }^{\text {opt }}$ differ from what we expect as illustrated in Fig. 2a: the reflection responses are jointly shifted in frequency by $\Delta f$. This can be eliminated by redesigning the element so that its center frequency is moved from $f_{0}$ to $f_{0}-\Delta f$. The optimization (cf. Subsection 2.1) results in a new element geometry $\boldsymbol{x}_{g}{ }^{1}$.

In the next step, the array model $\boldsymbol{R}_{f}$ is evaluated at the element geometry $\boldsymbol{x}_{g}{ }^{1}$ and the optimized phase taper $\boldsymbol{x}_{p}{ }^{\text {opt }}$. At this point, the active reflection coefficient responses are 
centered around $f_{0}$ at the $-10 \mathrm{~dB}$ level ( $c f$. Fig. 3). The entire design process requires only four high-fidelity simulations of the antenna array model. The computational cost of other operations is low and can be neglected compared with the cost of EM simulations.

a)

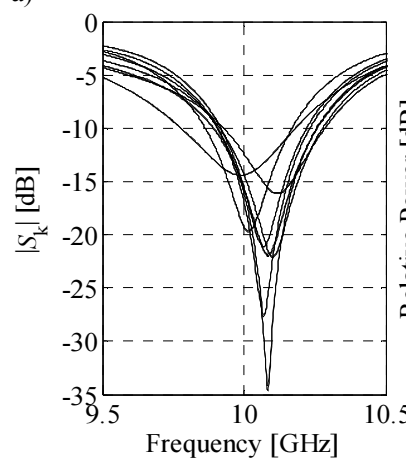

b)

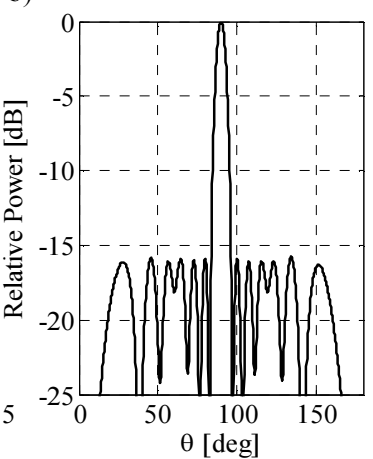

Fig. 2. The initially optimized EM array model $\boldsymbol{R}_{f}$ : a) The active reflection coefficients at all ports with the frequency shift $\Delta f$ denoted; b) the E-plane radiation pattern.

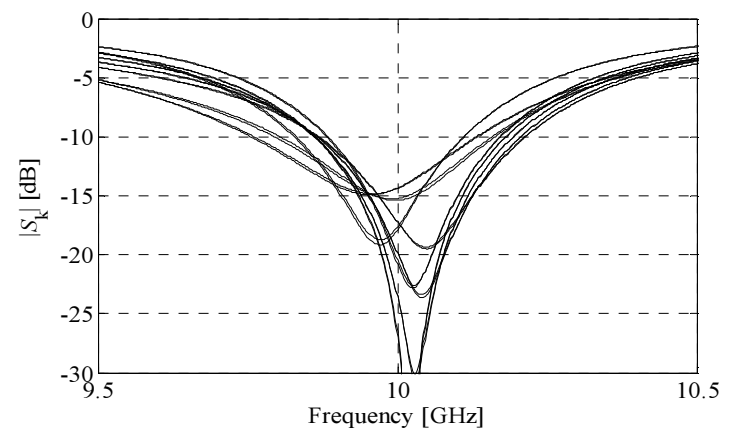

Fig. 3. The reflection responses (centered around $10 \mathrm{GHz}$ at the $-10 \mathrm{~dB}$ level) of the EM array model $\boldsymbol{R}_{f}$ with re-optimized element $\boldsymbol{x}_{g}{ }^{1}$.

Subsequently, the phase excitation tapers for the scanned array are found by iterative identification of the response correction term (4) and surrogate-assisted optimization (5), (6). This is executed, separately, for each scan angle $\alpha^{(k)}$ as follows (here, $\boldsymbol{x}_{p}{ }^{\text {opt.k }}$ is the optimized phase taper corresponding to $\alpha^{k}$ ):

1. Evaluate the EM array model $\boldsymbol{R}_{f}$ at $\boldsymbol{x}_{p}{ }^{\text {opt.k-1}}$ (progressive phase shift for $\alpha^{(k)}$ is applied separately);

2. Calculate the correction term $D(c f .(4))$;

3. Find the phase taper $\boldsymbol{x}_{p}^{\text {opt.k }}$ by optimizing the surrogate $\boldsymbol{R}_{s}(5)$ for the minimum SLL (corresponding to $\left.\alpha^{(k)}\right)$ using (6).

The above procedure requires only one evaluation of the high-fidelity model $\boldsymbol{R}_{f}$ per a scan angle.

\section{Case study: 16-element linear phased array}

Consider a linear array of microstrip antennas shown in Fig. 4. We search for excitation phases (phase taper) of signals, which are incident at the elements' inputs to minimize the Side- 
Lobe Level (SLL) in the E-plane. The active reflection coefficient responses of the array should be centered around the operational frequency of $10 \mathrm{GHz}$. Excitation amplitudes are uniform; center-to-center element spacing is a half wavelength.

\subsection{Element optimization}

An element (Fig. 4b) is implemented on a $0.76 \mathrm{~mm}$ thick layer of Taconic RF-35 [14]. The parameters are: the patch size is $d_{1}$ by $d_{2}$; the metal ground has the slot aperture of $w_{1}$ by $u_{1}$, the slot center is $v_{1}$ relative to the patch center; a feed substrate is another $0.76 \mathrm{~mm}$ layer of RF-35; the input $50 \mathrm{ohm}$ microstrip has the width of $w_{0}=1.7 \mathrm{~mm}$, the open end stub of length $v_{2}$ terminates the feed and $w_{c}$ is the chamber length of the input microstrip. The antenna was simulated in CST Microwave Studio [15].

The design variable vector is $\boldsymbol{x}_{g}=\left[\begin{array}{lllllll}d_{1} & d_{2} & w_{1} & u_{1} & v_{1} & v_{2} & w_{c}\end{array}\right]^{T}$. The design was carried out as described in Subsection 2.1. We use two EM models: the coarse-mesh model $\boldsymbol{R}_{e c}$ (70,000 mesh cells, the simulation time $30 \mathrm{~s})$ and the fine model $\boldsymbol{R}_{e f}(\sim 1,000,000$ cells, $10 \mathrm{~min})$. The initial design is $\boldsymbol{x}^{\text {(ini) }}=\left[\begin{array}{lllllll}9.0 & 7.4 & 2.0 & 6.0 & 2.5 & 6.0 & 1.5\end{array}\right]^{T} \mathrm{~mm}$. The optimized design $\boldsymbol{x}_{g}{ }^{\text {opt }}=\left[\begin{array}{llll}9.53 & 7.29 & 2.90\end{array}\right.$ $\left.\begin{array}{llll}5.50 & 1.91 & 5.50 & 1.48\end{array}\right]^{T} \mathrm{~mm}$ was found in 3 iterations at the total cost corresponding to about 12 simulations of $\boldsymbol{R}_{e f}$ (including 160 evaluations of $\boldsymbol{R}_{e c}$, which corresponds to the CPU time of about 80 minutes, and four evaluations of the high-fidelity model, or 40 minutes). The reflection response of this design with the $3.5 \%$ bandwidth at the $-10 \mathrm{~dB}$ level is centered at $10 \mathrm{GHz}$.

\subsection{Optimal excitation taper for main beam pointed broadside}

At this stage we search for a set of excitation phases $\left\{\alpha_{n}\right\}_{n=1, \ldots, N}$ of the linear array (Fig. 4a), so that the SLL of the array power pattern is minimized ( $c f$. Fig. 5).

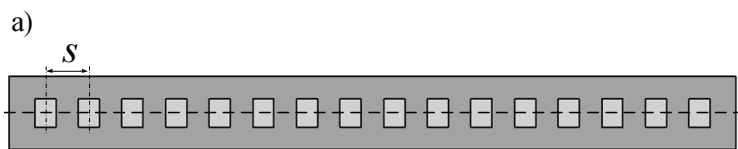

b)

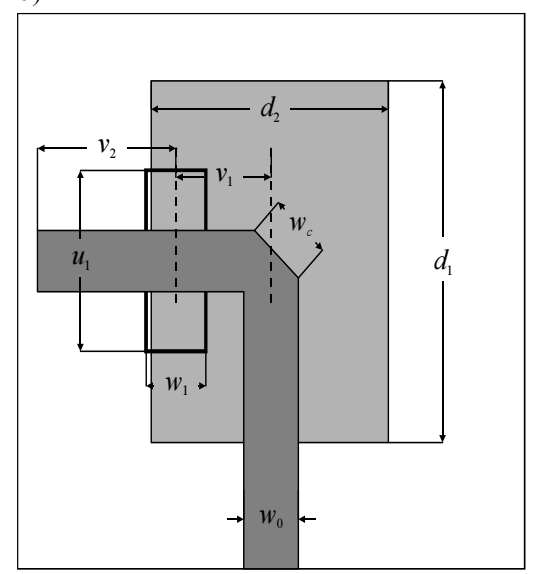

Fig. 4. A linear array of microstrip antennas: a) equally spaced array, the E-plane of the antenna array is shown with the dash-line; b) the top view of an array element (slot fed microstrip patch antenna); dielectric layers and the ground plane are shown transparent; the edges of the patch (light gray), contours of the slot (bold rectangle), open end micro-strip stub, and input micro-strip (dark gray) are shown with solid lines. 
We assume symmetrical phase excitations with respect to the array center: $\alpha_{N+1-n}=\alpha_{n}, n=$ $1, . . N / 2$; in this work $N=16$. The array spacing $s$ and amplitudes are uniform. The design variable vector was $\boldsymbol{x}_{p}=\left[\begin{array}{llll}\alpha_{1} & \alpha_{2} & \ldots & \alpha_{8}\end{array}\right]^{T}$. The uniform amplitude and phase array (SLL $\approx$ $-13.2 \mathrm{~dB})$ is the initial design, $\boldsymbol{x}_{p}^{(i n i)}=\left[\begin{array}{lllllll}0 & 0 & 0 & 0 & 0 & 0 & 0\end{array}\right]^{T}$ degrees. The CST high-fidelity model of the entire array $\boldsymbol{R}_{f}(\sim 10,000,000$ mesh cells, the evaluation time 76 minutes) was simulated for the power pattern. The array was optimized as in Subsections 2.2-2.3. The optimal phase taper for the main beam pointed broadside $\left(\mathrm{SLL}=-16.0 \mathrm{~dB}\right.$ ) was found at $\boldsymbol{x}_{p}{ }^{\text {opt }}=\left[\begin{array}{ll}-58.1 & 0.0\end{array}\right.$ $-95.9-70.7-71.4-64.4-65.6-59.9]^{T}$ degrees. The reflection responses at this design are shifted towards higher frequencies as shown in Fig. 2a. The re-optimized element design was found at $\boldsymbol{x}_{g}{ }^{1}=\left[\begin{array}{llllllll}9.38 & 7.34 & 2.9 & 5.5 & 1.91 & 5.5 & 2.05 & 1.28\end{array}\right]^{T} \mathrm{~mm}$. The fine-tuned optimal design

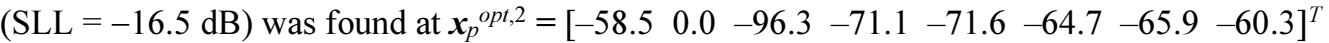
degrees by repeating (6) with the updated surrogate. Each execution of (6) takes a few seconds.

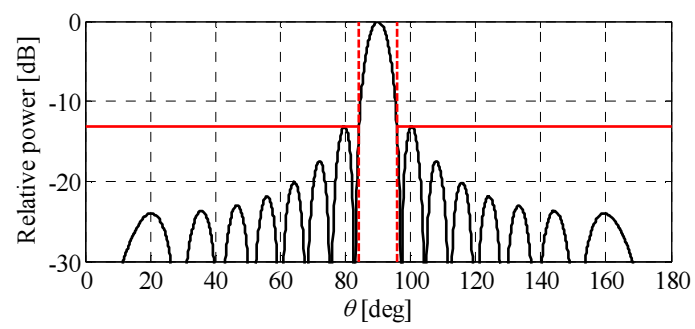

Fig. 5. The radiation pattern of an antenna array with design specifications shown as thick horizontal lines. The vertical dashed lines define the main beam sector.

a)

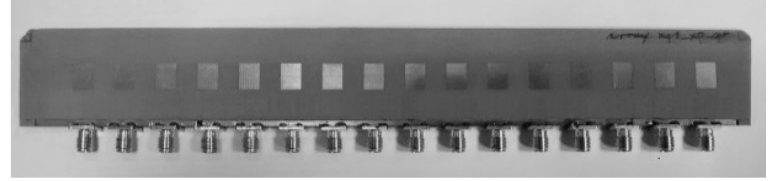

b)

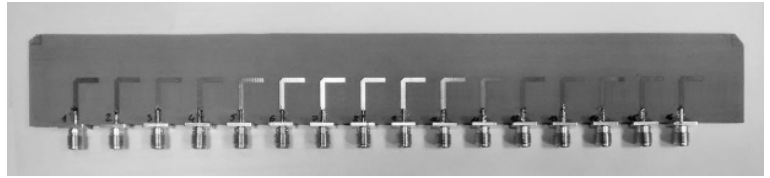

Fig. 6. The photograph of the manufactured array microstrip antennas: a) top; b) bottom.

\subsection{Array Optimisation for Scanning}

The procedure of Subsection 2.4 has been applied to find the optimum tapers for the scan angles of $5,10, \ldots$, and 50 degrees. The results are listed in Table 1. It should be noted, however, that the total phase excitation applied to a particular element for a given scan angle is the sum of the listed optimal value and the scan-dependent progressive phase shift [1] (not listed in Table 1). 


\subsection{Experimental Validation of the Optimal Design}

The final design with geometry of $\boldsymbol{x}_{g}^{\text {opt }}$ has been manufactured (the photograph shown in Fig. 6). The radiation patterns of this array, sixteen in total, with only one input fed at a time (i.e. one element active at a time) - while other terminated on matched loads - have been measured. The measured radiation patterns are shown in Fig. 7. The measured radiation patterns have been combined with the optimal phase tapers $\boldsymbol{x}_{p}{ }^{o p t, 2}$ and the progressive phase shifts to evaluate the array radiation pattern for different scan angles. The radiation patterns for the scan angles of zero and 40 degrees are shown in Fig. 8 (dot-lines). Excellent agreement of the simulated and evaluated (configured from the measured data) patterns was observed within \pm 50 degree sector of scan with the phase tapers listed in Table 1.

\subsection{Optimal Design as the Phased Antenna Array}

Appropriate interpolation enables to use the results of Table I for any angle within the scan sector. To evaluate performance of the linear array with the interpolated phase tapers at the high-fidelity level of description, the superposition model of the entire structure has been configured from sixteen high-fidelity discrete simulations, as described in [12].

The radiation and reflection responses of the array have been evaluated versus the scan angle, using the superposition model. The following excitations have been compared: the interpolated set of the optimal phase excitation tapers, the phase excitation taper obtained as an optimum for the zero scan angle and the uniform excitation. The excitations have been compared in the following figures: the SLL (the optimized figure) illustrated in Fig. 9; the achieved peak realized gain scan loss shown in Fig. 10; the beam broadening; and the total reflected power provided in Fig. 11.

Figure 9 clearly indicates that the interpolated set of the optimal phase excitation tapers is superior over the other excitations as it enables keeping the SLL at much lower levels within the wide sector of scans. This also justifies the optimization step described in Subsection 2.3 and reliability of interpolation of the optimal phase sets. At the same time, Fig. 9 shows that with the excitation being optimal at broadside, the SLL quickly degrades with the scan angle. The achieved realized gain scan losses ( $c f$. Fig. 10) are very close for all excitations up to the scan angles of \pm 50 degrees although the interpolated set of the optimal phase excitation tapers provides slightly lower scan losses. The beam broadening with all excitations is closely follows the large array limit [1]. For all excitations, the total reflected power, plotted in Fig. 11, stays under $-15 \mathrm{~dB}$ level almost up to the \pm 50 degrees of scan angles.

\section{Conclusion}

A technique for simulation-driven design of phased linear antenna arrays including the main beam scanning has been presented. Our approach produces phase-only excitation tapers with low side-lobes within the scan sector. Importantly, possible interactions and coupling within the structure are taken into account at the high-fidelity level of description due to the use of discrete EM simulations. The use of surrogate-based optimization and the proposed array factor correction functions enables to keep the design costs at a low level. The proposed method has been verified experimentally through physical measurements of the manufactured array prototype. The comparison of the objective figures (in this work, the SLL) justifies using the presented approach to avoid degradation of the radiation pattern in the process of scan. 

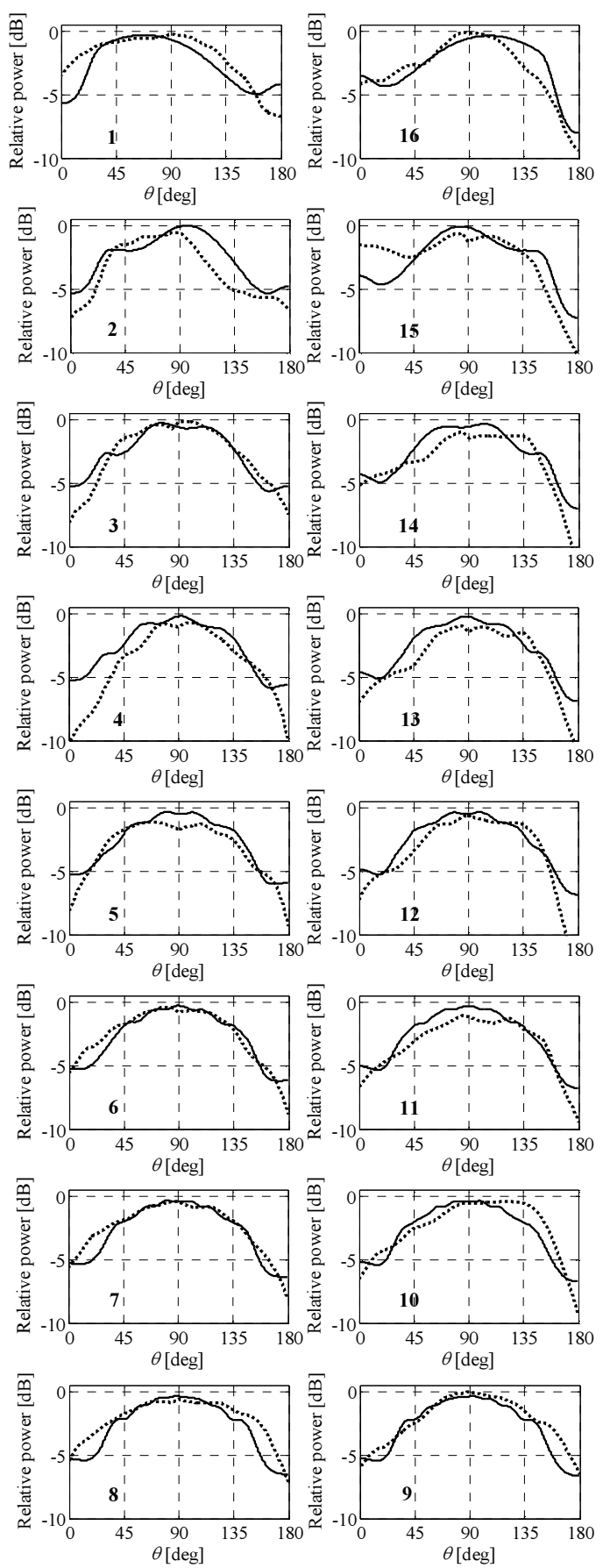

Fig. 7. The antenna array patterns with one element active. The measured $(\cdot \boldsymbol{*})$ and simulated $(-)$ patterns are normalized, respectively, to the maximum measured and simulated peak values.

Element numbers are listed in the bottom-left corners. 
a)

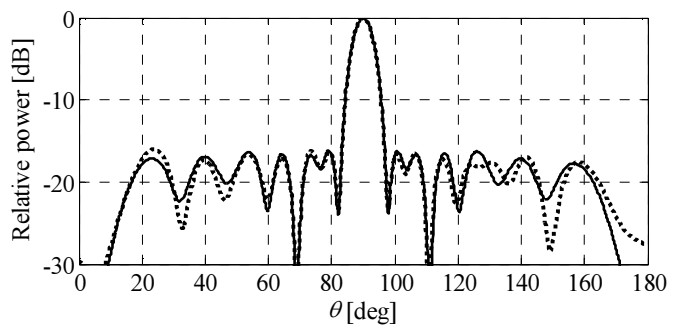

b)

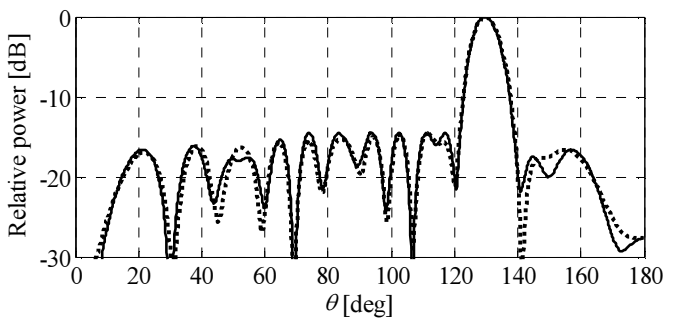

Fig. 8. The radiation pattern of the final design of geometry $\boldsymbol{x}_{g}{ }^{o p t}$ with optimal phase tapers in the E-plane at $10 \mathrm{GHz}$ simulated with the high-fidelity array model $\boldsymbol{R}_{f, a}(-)$; evaluated using 16 measured radiation patterns of the array with only one element active (-*): a) for the zero scan angle (phases corresponding to the scan angle of 0 deg. in Table 1); b) for the scan angle of 40 degrees (phases corresponding to the scan angle of 40 deg. in Table 1).

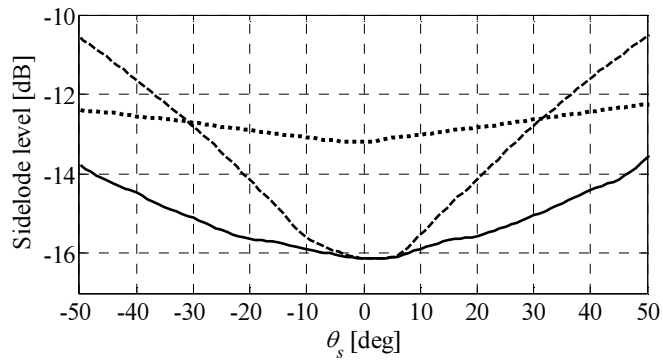

Fig. 9. The side-lobe level versus the scan angle: the interpolated set of the optimal phase excitation tapers $(-)$ the phase excitation taper obtained as an optimum for the zero scan angle $(--)$, the uniform excitation (--).

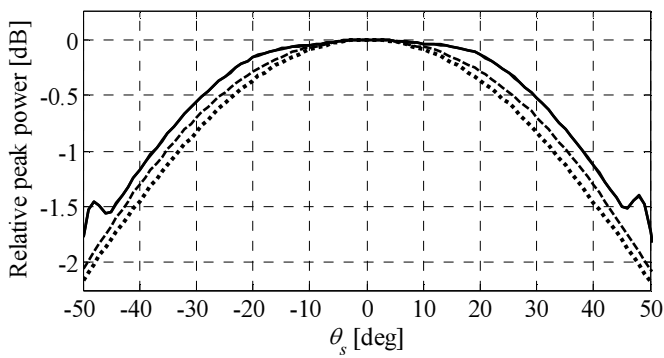

Fig. 10. The simulated achieved peak realized gain scan loss (relative to that at the zero scan angle which is 18 $\mathrm{dB}$ for the uniform excitation and $17 \mathrm{~dB}$ for the optimized excitation): the interpolated set of the optimal phase excitation tapers (-); the phase excitation taper obtained as an optimum for the zero scan angle $(--)$; the uniform excitation $(\boldsymbol{\cdot \cdot})$. 
Table 1. The optimal Phase Tapers*.

\begin{tabular}{|c|c|c|c|c|c|c|c|c|}
\hline \multirow{2}{*}{$\begin{array}{c}\text { Scan } \\
\left.\text { Angle [ }{ }^{\circ}\right]\end{array}$} & \multicolumn{8}{|c|}{ Elements } \\
\cline { 2 - 9 } & $1(16)$ & $2(15)$ & $3(14)$ & $4(13)$ & $5(12)$ & $6(11)$ & $7(10)$ & $8(9)$ \\
\hline 0 & -58.5 & 0.0 & -96.3 & -71.1 & -71.6 & -64.7 & -65.9 & -60.3 \\
\hline 5 & -51.8 & 0.0 & -97.0 & -67.7 & -70.2 & -67.5 & -61.4 & -61.5 \\
\hline 10 & -48.6 & 0.0 & -95.9 & -64.1 & -70.6 & -63.7 & -60.9 & -59.1 \\
\hline 15 & -44.6 & 0.0 & -92.4 & -62.6 & -67.5 & -60.6 & -57.6 & -55.4 \\
\hline 20 & -40.4 & 0.0 & -88.7 & -60.7 & -64.7 & -58.9 & -55.6 & -53.6 \\
\hline 25 & -37.7 & 0.0 & -87.1 & -60.8 & -63.3 & -58.4 & -54.7 & -52.9 \\
\hline 30 & -36.1 & 0.0 & -86.4 & -61.3 & -62.2 & -58.1 & -53.8 & -52.0 \\
\hline 35 & -34.7 & 0.0 & -85.8 & -62.0 & -61.7 & -58.4 & -53.5 & -51.9 \\
\hline 40 & -33.7 & 0.0 & -85.7 & -62.8 & -61.1 & -58.4 & -52.7 & -51.2 \\
\hline 45 & -32.8 & 0.0 & -85.1 & -63.6 & -61.0 & -59.2 & -53.1 & -51.9 \\
\hline 50 & -32.6 & -48.3 & -78.2 & 0.0 & -38.5 & -38.9 & -46.1 & -38.9 \\
\hline
\end{tabular}

${ }^{*}$ The listed values do not include the progressive phase shift.

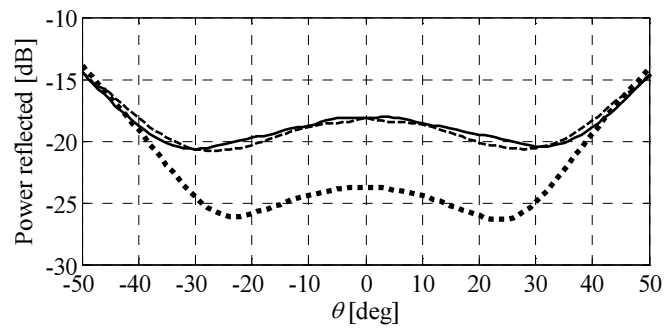

Fig. 11. The simulated total reflected power versus the scan angle: the interpolated set of the optimal phase tapers (-), the phase excitation taper obtained as an optimum for the zero scan angle $(--)$, the uniform excitation $(\boldsymbol{- \cdots})$.

\section{Acknowledgments}

The authors would like to thank Computer Simulation Technology AG, Darmstadt, Germany, for making CST Microwave Studio available. This work was supported in part by the Icelandic Centre for Research (RANNIS) under grant no. 141272051.

\section{References}

[1] Mailloux, R.J. (2005). Phased Array Antenna Hanbook. Artech House.

[2] Amitay, N., Butzien, P., Heidt, R. (1968). Match optimization of a two-port phased array antenna element. IEEE Transactions on Antennas Propag., 16(1), 1947-57.

[3] De Ford, J.F., Gandhi, O.P. (1988). Phase-only synthesis of minimum peak sidelobe patterns for linear and planar arrays. IEEE Trans. Antennas Prop., 36(2), 191-201.

[4] De Ford, J.F., Gandhi, O.P. (1988). Mutual coupling and sidelobe tapers in phase-only antenna synthesis for linear and planar arrays. IEEE Trans. Antennas Prop., 36(11), 1624-1629.

[5] Haupt, R.L. (1995). Optimum quantized low sidelobe phase tapers for arrays. Electronics Lett., 31(14), 1117-1118

[6] Bray, M.G., Werner, D.H. (2002). Optimization of Thinned Aperiodic Linear Phased Arrays Using Genetic Algorithms to Reduce Grating Lobes During Scanning. IEEE Transactions on Antennas Propag., 50(12), 1732-1742.

[7] Boeringer, D.W., Werner, D.H. (2004). Particle Swarm Optimization Versus Genetic Algorithms for Phased Array Synthesis. IEEE Transactions on Antennas Propag., 52(3), 771-779. 
[8] Yang, S.H., Kiang, J.F. (2014). Adjustment of Beamwidth and Side-Lobe Level of Large Phased-Arrays Using Particle Swarm Optimization Technique. IEEE Transactions on Antennas Propag., 62(1), 138-144.

[9] Chryssomallis, M.T., Christodoulou, C.G. (2004). A Circuit-Based Optimization Approach for Improving the Pattern of Uniform Array Antennas via Phase Control. IEEE Transactions on Antennas Propag., 52(10), 2776-2781.

[10] Kozieł, S., Ogurtsov, S. (2014). Antenna design by simulation-driven optimization. Springer.

[11] Kozieł, S., Ogurtsov, S. (2014). Simulation-Based Design of Microstrip Linear Antenna Arrays Using Fast Radiation Response Surrogates. IEEE Antennas Wireless Propag. Lett., DOI: 10.1109/LAWP.2014. 2377519.

[12] Koziel, S., Ogurtsov, S. (2014). Phase-spacing optimization of linear microstrip antenna arrays by EMbased superposition models. Proc. 2014 Loughboroh Antenna Propag. Conf. (LAPC), 26-30.

[13] Bandler, J.W., Cheng, Q.S., Dakroury, S.A., Mohamed, A.S., Bakr, M.H., Madsen, K., Søndergaard, J. (2004). Space mapping: the state of the art. IEEE Trans. Microwave Theory Tech., 52(1), 337-361.

[14] ORCER RF-35, Data Sheet, 2014, Taconic, 136 Coonbrook Rd., Petersburgh, N.Y. 12138, USA, http://www.taconic-add.com/pdf/rf35.pdf

[15] CST Microwave Studio, ver. 2013, CST AG, Bad Nauheimer Str. 19, D-64289 Darmstadt, Germany, 2013.

[16] Koziel, S., Ogurtsov, S. (2012). Linear antenna array synthesis using gradient-based optimization with analytical derivative. Proc. 2012 IEEE APS Intl. Sympos. 